\title{
Motivos de aderência das idosas incontinentes após follow-up de 24 meses de treinamento muscular
}

\author{
Reasons for adherence of incontinent elderly women after a follow-up of 24 \\ months of muscle training
}

Giovana Zarpellon Mazo ${ }^{1 *}$, Eduardo Capeletto ${ }^{1}$, Keyla Mara dos Santos ${ }^{1}$, Franciele da Silva Pereira $^{1}$, Enaiane Cristina Menezes ${ }^{1}$, Janeisa Franck Virtuoso ${ }^{2}$

ARTIGO ORIGINAL | ORIGINAL ARTICLE

\begin{abstract}
Objetivo: investigar os motivos de aderência 24 meses após intervenção com treinamento dos musculos do assoalho pélvico (TMAP) com e sem musculação em idosas com incontinência urinária (IU). Método: Após o follow-up de 24 meses das intervenções foram contatadas 23 idosas. Foram aplicados o International Consultation on Incontinence Questionnaire - Short Form (ICIQ-SF) para verificar a frequência e gravidade da IU e o Questionário de Motivos para a Prática do TMAP para descrever os motivos de aderência ao TMAP e a prática de exercícios físicos (AFR). Resultados: Após 24 meses do tratamento da IU por meio do TMAP com e sem a prática da musculação $30,4 \%(n=7)$ das idosas permaneceram continentes. A adesão ao TMAP foi de $52,2 \%(n=12)$ e ao AFR de $73,9 \%(n=17)$, após 24 meses da intervenção. O motivo para a prática do TMAP foi a prevenção da IU. Para a AFR, o principal motivo foi a melhora da saúde geral. Conclusão: Sugerem-se novos estudos que utilizem estratégias para facilitar a adesão ao tratamento, num período longo de seguimento.

Palavras-chave: incontinência urinária, idosas, exercício físico.
\end{abstract}

ABSTRACT

Objective: to investigate the adhesion motives 24 months after intervention with training of pelvic floor muscles (PFMT) with and without bodybuilding in elderly women with urinary incontinence (UI). Method: After the 24-month follow-up of the interventions, 23 elderly women were contacted. The International Consultation on Incontinence Questionnaire (ICIQ-SF) was used to verify the frequency and severity of UI and the TMAP Practice Reasoning Questionnaire to describe the reasons for adherence to PFMT and physical exercise practice (RPA). Results: After 24 months of UI treatment through TMAP with and without bodybuilding, $30.4 \%(n=7)$ of the elderly women remained continents. Adherence to TMAP was $52.2 \%(n=12)$ and AFR was $73.9 \%(n=17)$, after 24 months of intervention. The reason for the practice of PFMT was the prevention of UI. For RPA, the main reason was the improvement of general health. Conclusion: We suggest new studies that use strategies to facilitate adherence to treatment, over a long period of follow-up.

Keywords: urinary incontinence, elderly, physical exercise.

\footnotetext{
${ }^{1}$ Universidade do Estado de Santa Catarina, Florianópolis-SC, Brasil

${ }^{2}$ Universidade Federal de Santa Catarina, Aranguá-SC, Brasil

* Autor correspondente: giovana.mazo@udesc.br
} 


\section{INTRODUÇÃO}

A Incontinência Urinária (IU) é definida como qualquer perda involuntária de urina (Haylen et al., 2010) e a sua maior ocorrência ocorre no sexo feminino $(28,2 \%)$ (Tamanini, 2017) e com o aumento da idade (Marques et al., 2015). Sabese que o treinamento dos músculos do assoalho pélvico (TMAP) é fortemente recomendado para o tratamento da IU (Dumoulin et al., 2016). No entanto, sua efetividade depende da adesão das pacientes à realização dos exercícios propostos pelo profissional de saúde (BØ \& Herbert, 2013).

Em uma revisão sistemática, realizada para avaliar o resultado a longo prazo do TMAP para a IU, foi possível observar que as perdas urinárias a longo prazo (um ano após o término da intervenção) variaram entre $0 \%$ e $39 \%$ e que a melhora dos sintomas a curto prazo do TMAP pode ser mantido em seguimento a longo prazo, apesar da heterogeneidade entre os estudos (BØ \& Hilde, 2012).

Destaca-se ainda a importância da manutenção da atividade física regular (AFR) nas idosas (Morrisroe, 2014), pois os baixos níveis de atividade física têm sido relacionados ao aparecimento dos sintomas de IU nesta população (Menezes, Virtuoso \& Mazo, 2015).
Portanto, torna-se necessário conhecer os motivos que facilitam e dificultam a adesão ao TMAP e à prática de AFR a longo prazo, visando auxiliar na manutenção dos resultados adquiridos no tratamento para IU. Dessa forma, pretende-se investigar os motivos de aderência do TMAP e da prática de AFR, após 24 meses de intervenção com treinamento dos músculos do assoalho pélvico (TMAP) com e sem musculação em idosas com incontinência urinária (IU).

\section{MÉTODO}

Trata-se de um estudo descritivo transversal após um follow-up de 24 meses.

\section{Participantes}

A 23 idosas que foram submetidas previamente a um ensaio clínico randomizado e que pertenciam ao Grupo Intervenção (GI) que recebeu o TMAP associado à musculação $(\mathrm{n}=10)$ e ao grupo controle (GC) que realizou apenas o TMAP $(n=13)$. Para este este estudo foram excluídas as participantes do ensaio clínico que foram submetidas a qualquer intervenção cirúrgica na região do assoalho pélvico ou realizado qualquer tratamento para IU antes do 24 meses (Figura1).

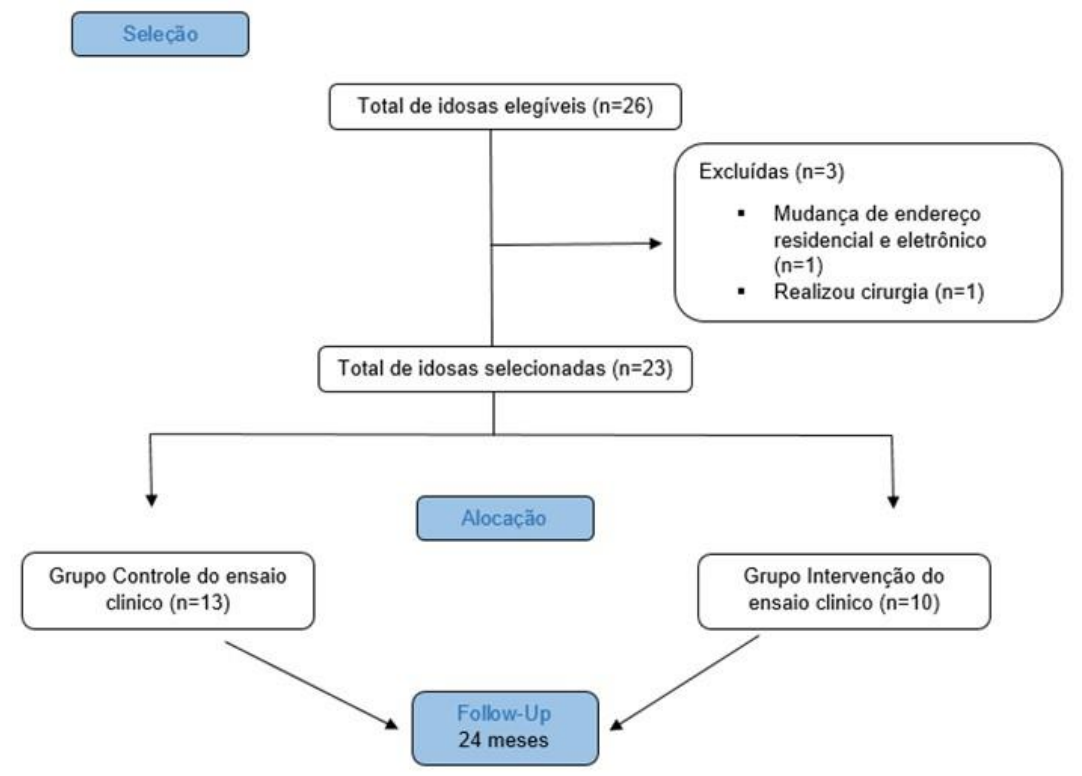

Figura 1. Diagrama representando a seleção e a alocação das idosas após 24 de Follow-Up do ensaio clínico

\section{Instrumentos e Procedimentos}

O estudo foi aprovado pelo Comitê de Ética Envolvendo Seres Humanos (CEPSH) da
Universidade do Estado de Santa Catarina (UDESC) sob CAAE no 14957313.9.0000.01180 e cadastrado no Registro Brasileiro de Ensaios 
Clínicos (ReBEC), vinculado ao Ministério da Saúde sob o no U1111-1149-2398.

Foram recolhidas informações do estudo prévio (VIRTUOSO, 2015), correspondentes ao início das intervenções, como o endereço e telefone das idosas. Após o follow-up de 24 meses do ensaio clínico todas as idosas foram contatadas pelos pesquisadores, independente da cura da IU. Foram aplicados, via contato telefônico, dois questionários: International Consultation on Incontinence Questionnarie - Short Form (ICIQ-SF), para verificar a presença e a gravidade da IU (Tamanini, 2004) e o Questionário de Motivos para a Prática do Treinamento dos Músculos do Assoalho Pélvico (TMAP) e Atividade Física Regular (AFR), elaborado pelos pesquisadores, para identificar se as idosas continuavam praticando o TMAP e AFR e quais os motivos.

\section{Análise estatística}

Foi realizada estatística descritiva (medidas de posição e dispersão e frequência simples e relativa), utilizando SPSS, versão 20.0. Para verificar os motivos para a prática do TMAP e AFR, realizou-se análise de conteúdo temática (Minayo, 2013), por meio do Software Maxqda (Verbi, Alemanha).

\section{RESULTADOS}

A média de idade das idosas participantes deste estudo foi de $67,96 \pm 4,76$ anos. Dentre as 23 idosas, 69,6\% $(n=16)$ voltaram a perder urina 24 meses após o término das intervenções. Das idosas incontinentes, $10(62,5 \%)$ pertenciam ao
GC (apenas TMAP), 9 (56,3\%) não permaneceram praticando TMAP a domicilio. Dentre as 16 idosas incontinentes, e $11(68,7 \%)$ continuaram praticando AFR, mesmo com sintomas de IU. No entanto, 30,4\% $(n=7)$ das idosas apresentaram manutenção da continência urinária 24 meses após receberem o tratamento da IU por meio do TMAP com e sem a prática da musculação (Tabela1).

No geral, a adesão ao TMAP após 24 meses foi de $52,2 \%(n=12)$ e à AFR de $73,9 \%(n=17)$. O escore total do ICIQ-SF no início das intervenções foi, em média, de $11,70 \pm 3,0$ e no final, em média, de $2,78 \pm 3,7$. Após follow-up de 24 meses foi, em média, de 5,70 $\pm 5,2$.

A Figura 2 apresenta os principais motivos relatados pelas idosas para a continuação ou não da prática de TMAP e AFR após 24 meses das intervenções (GI e GC).

Tabela 1

Características das idosas continentes e incontinentes, após o follow-up de 24 meses do ensaio clínico

\begin{tabular}{lll}
\hline & $\begin{array}{c}\text { Continentes } \\
\mathrm{f}(\%)\end{array}$ & $\begin{array}{c}\text { Incontinentes } \\
\mathrm{f}(\%)\end{array}$ \\
\hline $\mathrm{N}^{\mathrm{o}}$ Idosas & $7(30,4)$ & $16(69,6)$ \\
\hline Grupos & $\mathrm{de}$ & \\
$\begin{array}{l}\text { Intervenções } \\
\quad \mathrm{GI}\end{array}$ & $3(42,9)$ & $6(37,5)$ \\
$\quad \mathrm{GC}$ & $4(57,1)$ & $10(62,5)$ \\
\hline Prática do TMAP & $5(71,5)$ & $7(43,8)$ \\
$\quad$ Sim & $2(28,5)$ & $9(56,3)$ \\
$\quad$ Não & & \\
\hline Prática de AFR & $6(85,7)$ & $11(68,7)$ \\
$\quad$ Sim & $1(14,3)$ & $5(31,3)$ \\
$\quad$ Não & &
\end{tabular}

Nota. TMAP $=$ Treinamento dos músculos do assoalho pélvico, $\mathrm{AFR}=$ atividade física regular, $\mathrm{GI}=\mathrm{Grupo}$ intervenção, $\mathrm{GC}=$ Grupo Controle.

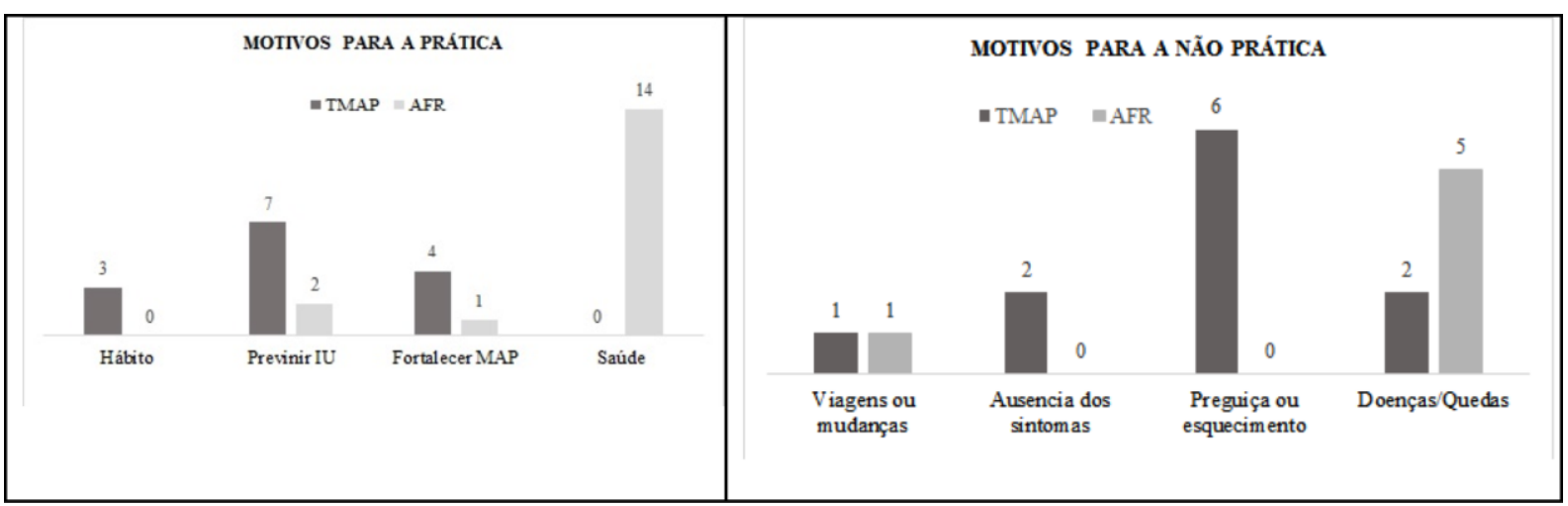

Figura 2. Motivos relatados pelas idosas para a continuação ou não da prática de TMAP e AFR após 24 meses das intervenções, onde: TMAP $=$ treinamento dos músculos do assoalho pélvico, $\mathrm{AFR}=$ atividade física regular, $\mathrm{IU}=$ incontinência urinária, $\mathrm{MAP}=$ músculos do assoalho pélvico. 


\section{DISCUSSÃO}

Os motivos que levaram as idosas a praticarem TMAP e AFR, após 24 das intervenções do TMAP com e sem a musculação, foram o hábito, a prevenção da IU, fortalecimento dos MAP e a saúde geral. Por outro lado, os motivos que impediram as idosas de continuarem a prática do TMAP e AFR após as intervenções foram as viagens ou mudanças, ausência de sintomas de IU, preguiça ou esquecimento e doenças ou quedas.

Observa-se no presente estudo que após o follow-up de 24 meses do ensaio clínico a taxa de cura da IU das idosas foi expressiva (30,4\%). Tal percentual é superior à taxa de cura apresentada no estudo de Plotti (2018), que foi de $24 \%$ após um follow-up de $8,3 \pm 3,5$ anos, em média, de um procedimento cirúrgico de implante de agentes de volume para correção da IU e, do estudo de Lsooes (2017) que verificou uma melhora de $28,3 \%$, após um follow up de 2 anos de uso de um aplicativo móvel para tratar a IU de esforço com foco no TMAP.

Em conformidade com a alta taxa de cura apresentada, no presente estudo percebe-se uma frequente preocupação entre as idosas em continuar a prática do TMAP, principalmente para prevenir a IU e fortalecer os MAP, como relatam as idosas: "Para não acelerar o processo da IU, para fortalecer essa parte" (MBV, 67 anos); "Por precaução, aprendi que deveria fazer" (AL, 71 anos); "Fortalecer os músculos do assoalho pélvico" (MTB, 65 anos); e, "Senti que fez bem na época, sinto necessidade de ter mais tônus muscular" (MNR, 68 anos).

Esse resultado mostra que a prática do TMAP pelas idosas continentes foi motivada pela manutenção dos resultados adquiridos no tratamento. Além disso, quando inicialmente bem orientadas sobre a situação, as idosas são capazes de manter a atividade adequada a longo prazo com intuito de prevenir disfunções e promover a própria saúde. Segundo Santacreu e Fernandez-Ballesteros (2011), a manutenção dos resultados de um tratamento para IU por meio do TMAP, só seria mantida a longo prazo, caso a prática do TMAP fosse mantida.
Em relação à manutenção da prática de AFR, as idosas deste estudo a atribuíram como fator importante para melhorar da saúde geral, conforme os seguintes relatos: "Saúde, sou hipertensa, para ter qualidade de vida" (MBV, 67 anos); "Tudo de bom, para a saúde para não ficar com dores" (MAV, 64 anos); e "Melhorar a condição física, para ir e vir" (LN, 66 anos).

Vale salientar que é necessário criar o hábito de praticar os exercícios regularmente para que os resultados do TMAP sejam mantidos, sendo que a adesão terapêutica é o fator prognóstico mais importante (Kruger, Luz \& Virtuoso, 2011). A AFR pode ser considerada um fator protetor da IU (Menezes, Virtuoso \& Mazo, 2015).

O acompanhamento do tipo de atividade física realizada pelas idosas ao longo dos 24 meses da intervenção foi uma limitação do estudo. Destacase que este estudo avança em termos de um follow-up de 24 meses.

\section{CONCLUSÕES}

Os motivos que levaram as idosas a praticarem TMAP e AFR, após 24 das intervenções do TMAP com e sem a musculação, foram o hábito, a prevenção da IU, fortalecimento dos MAP e a saúde geral. A taxa de cura da IU das idosas foi expressiva $(30,4 \%)$. Assim, torna-se importante a elaboração de estratégias para que as idosas sigam com a prática do TMAP e de atividade física regular, visando a manutenção da saúde e da diminuição dos sintomas de IU após o tratamento.

\footnotetext{
Agradecimentos:

A CAPES pela bolsa de pesquisa e ao CNPQ pela bolsa de produtividade.
}

\section{Conflito de Interesses:}

Nada a declarar.

Financiamento:

Nada a declarar. 


\section{REFERÊNCIAS}

Bø, K., \& Herbert, R. D. (2013). There is not yet strong evidence that exercise regimens other than pelvic floor muscle training can reduce stress urinary incontinence in women: a systematic review. Journal of Physiotherapy, 59, 159-168.

Bø, K., \& Hilde, G. (2012). Does it work in the long term? A systematic review on pelvic floor muscle training for female stress urinary incontinence. Neurourology and Urodynamics, 32(3), 215-223. doi:10.1002/nau.22292

Dumoulin, C., Hay-Smith, E. J. C., \& Mac habéeSéguin, G. (2016). Pelvic floor muscle training versus no treatment, or inactive control treatments, for urinary incontinence in women. The Cochrane Library.

Haylen, B. T., De Ridder, D., Freeman, R. M., Swift, S. E., Berghmans, B. L. J. (2010). An International Urogynecological Association (IUGA)/International Continence Society (ICS) joint report on the terminology for female pelvic floor dysfunction. Neurourology and urodynamics, $29(1), 4$.

Kruger, A., Luz, S., \& Virtuoso, F. J. (2011). Home exercises for pelvic floor in continent women one year after physical therapy treatment for urinary incontinence: an observational study. Revista Brasileira de Fisioterapia, 15(5), 351-356.

Lsooes, V. H. (2017). Self-management of stress urinary incontinence via a mobile app: two-year follow-up of a randomized controlled trial. Acta Obstetricia et Gynecologica Scandinavica, 96(10), 1180-7

Marques, L. P. (2015). Demographic, health conditions, and lifestyle factors associated with urinary incontinence in elderly from Florianópolis, Santa Catarina, Brazil. Revista Brasileira de Epidemiologia, 18(3), 595-606.
Menezes, E. C., Virtuoso, J. F., \& Mazo, G. Z. (2015). Older women with urinary incontinence present less physical activity level usual. Revista Brasileira de Cineantropometria e Desempenho Humano, 17(5), 612-620.

Minayo, M. C. S. (2013). O desafio do conhecimento: pesquisa qualitativa em saúde. (13 ${ }^{\mathrm{a}}$. ed). São Paulo: Hucitec.

Morrisroe, S. N. (2014). Correlates of 1-Year Incidence of Urinary Incontinence in Older Latino Adults Enrolled in a Community-Based Physical Activity Trial. Journal of the American Geriatrics Society, $62(4), 740-746$.

Plotti, F. M. R. (2018). Long-term follow-up of bulking agents for stress urinary incontinence in older patients. Menopause.

Santacreu, M., \& Fernández-Ballesteros, R. (2011). Evaluation of a behavioral treatment for female urinary incontinence. Clinical Interventions in Aging, 6, 133-139.

Tamanini, J. T. N. (2004). Validação para o português do "Internacional Consultation on Incontinence Questionnarie - Short Frem" (ICIQ-SF). Revista da Saúde Pública, 38(3), 438-444.

Tamanini, J. T. N. (2017). A populational-based survey on the prevalence, incidence, and risk factors of urinary incontinence in older adultsresults from the "SABE STUDY". Neurourology and Urodynamics. 37(1), 466-477. doi: $10.1002 /$ nau. 23331

Virtuoso, J. F. (2015). Efeito da prática de musculação associada ao treinamento dos músculos do assoalho pélvico em mulheres idosas com incontinência urinária: Um ensaio clínico randomizado. (Tese de Doutorado em Ciências do Movimento Humano) Universidade do Estado de Santa Catarina, Florianópolis, Brasil.

Todo o conteúdo da revista Motricidade está licenciado sob a Creative Commons, exceto quando especificado em contrário e nos conteúdos retirados de outras fontes bibliográficas. 